\title{
State of charge estimation for electric vehicle battery based on amended Ah metrology
}

\author{
Qiang Zhao ${ }^{1, *}$, Cheng-Jun Shao ${ }^{1}$ and Ying-Hua Han $^{2}$ \\ ${ }^{I}$ College of Information Science and Engineering, Northeastern University, Shenyang, \\ 110000, China \\ ${ }^{2}$ School of Computer and Communication Engineering, Northeastern University at \\ Qinhuangdao, Qinhuangdao,066004,China \\ E-mail: zhaoqiang@neuq.edu.cn
}

\begin{abstract}
The battery is the power source of electric vehicles and its performance has a direct influence on the power performance and the trip range. The state of charge (SOC) of the battery is one of the most important parameters in the use process of a battery. At the same time, the estimation accuracy of SOC can prevent battery over charging or over discharging, extending the service life of the battery effectively, and forecasting the remainder range accurately while traveling. The estimation of SOC is a very complicated work, due to the high nonlinear of the process of estimating. Moreover, SOC is affected by many factors, such as temperature, charge and discharge efficiency and aging factors and so on. In this paper, the parameters which affect the estimation accuracy of SOC are analyzed. To improve the estimation accuracy of SOC, an amended model is proposed, which combines Ah Metrology and open-circuit voltage with the correction on charge and discharge efficiency, aging factors, the initial SOC and capacity of battery. Simulation results show that an amended model of Ah Metrology can improve the estimation accuracy of SOC and reduce the error, which validates the feasibility and reliability of the proposed method.
\end{abstract}

Keywords: Electric Vehicle; Lithium-ion Battery; SOC; Ah Metrology.

\section{Introduction}

The rapid development of automobile industry not only pushed forward the world economy and transportation development, but also brought a series of problems, especially the negative impact of increased demand for resources and the environment. In order to meet the challenges of environmental and energy problem, the automotive industry is facing a revolutionary change. The electric vehicle on the environmental protection and energy saving has unparalleled advantages [1], including reduced dependency on nonrenewable energy resources, lowering of $\mathrm{CO}_{2}$ emissions and leading a lower carbon lifestyle. 
The battery is the core of the electric vehicle and its performance affects the power performance and the trip range directly. SOC is an important indicator for the battery. So SOC estimation accuracy is one of the most significant aspects of the battery. Accurate SOC estimation has a lot to do with the range of a electric vehicle. In the regard, researchers have studied the SOC estimation actively and achieved certain results. Accurate estimation of SOC is a challenge. A lot of SOC estimation methods are proposed, including Ah Metrology, the opencircuit voltage method, internal resistance method, Kalman filtering method and some optimized intelligent method and so on [2]. Kalman filter is a linear quadratic estimation algorithm which has advantages of converging quickly and performing well with different initial values. However, Kalman filter assumes the noise as Gaussian white, which is not true for battery management, where voltage and current sensors often suffer with colored noises. Meanwhile, Kalman filter requires an accurate system model, which is unrealistic in practice. Research shows that neural networks, such as a BP network, can estimate SOC with a high accuracy, but the whole network can't be built without lots of experiment data. At the same time, it often takes a long time for learning process, leading to a high consumption of computing resources, which is not appropriate in embedded electric vehicle battery systems.

In [3], an adaptive model for simulating the voltage relaxation process is developed to predict the final static open-circuit voltage in a few minutes. Other model-based method with filter algorithm, such as extend Kalman filter, is introduced in this field to improve the accuracy of SOC estimationsby removing measurement noise [4]. The extend Kalman filter method is based on an equivalent circuit model and realises SOC estimation by means of establishing state-space representation.However, this method strongly depends on the precision of the battery model and the system noise. In [5], it put forward an improved ampere-hour integral method to estimate SOC and analyze the parameters which affect the estimation accuracy of integral method. In [6], the lithium-ion battery model parameters are identified to get a more accurate and robust SOC estimation using an adaptive unscented Kalman filtering method. An adaptive adjustment of the noise covariances in the estimation process is implemented using a technique of covariance matching in the unscented Kalman filter context. In [7], an efficient electrochemical model-based SOC estimation algorithm is proposed, which uses electrochemical models to enable an accurate estimation of SOC as well as during high current events. In [8, 9], a method which is suitable for different temperature conditions is proposed. This algorithm mainly has considered the available capacity variation of battery at different temperature for SOC estimation. Ah Metrology,due to the circuit 
structure is easy to achieve, has become the most widely used estimation method. The Ah counting approach, is the basic approach followed directly by the definition of SOC and also the simplest. This method is accurate only if the current transducer is fairly precise and sufficient SOC recalibration points are available.

The existence of some factors, such as temperature, aging factors, the inaccuracy of the current, which has resulted in accumulation of error, has made the estimation accuracy of SOC low. So, in this paper, we put forward a model,combined open-circuit voltage method and Ah Metrology. Through opencircuit voltage method, the SOC initial value is obtained and used to improve the estimation accuracy of SOC, capacity of battery, aging factors, charging and discharging efficiency are all corrected.

The remainder of this paper is organized as follows. Section 2 describes mathematical modeling of an electric vehicle battery. The proposed estimate model of an amendment Ah method is presented in Section 3. Simulation results are given in Section 4. Section 5 concludes this paper.

\section{Mathematical Modeling of a Battery}

It is difficult to obtain a battery model since Li-ion batteries are considered to be complex electrochemical and nonlinear systems. Attempts have been made to evaluate the models for the estimation of Li-ion batteries, such as Rint Model [10], the first-order RC model [11], the second-order RC model [12], and the impedance model [13]. The estimation could be more accurate if the model can characterize the battery better, but it could also cause a more complex computation problem.

Theoretically, the standard remaining capacity is limited by the amount of active material in the electrolyte and electrodes. Knowledge of the specified current capacity motivates the following simple SOC estimation algorithm.

The percentage remaining SOC is calculated as

$$
S O C=100\left(\frac{Q_{c}-Q_{i}(t)}{Q_{c}}\right)
$$

where $Q_{c}$ is standard remaining capacity, and $Q_{i}(t)$ could be expressed as

$$
Q_{i}(t)=\int_{0}^{t} i(\tau) d \tau
$$

where $i(\tau)$ is the current which is positive when charging.

Such an ampere-hour method is very simple and concise, which could be implemented in practice easily, leading to a widely usage in the industry 
applications, in the portable electronics and also in electric vehicle industry. However, although this method is straight forward to implement, it has a few practical drawbacks. First, it requires the initial SOC known. Second, the algorithm assumes that the current capacity is a known fixed quantity. But the current capacity is time varying and dependent upon the current profile and temperature. The rated capacity is rarely achieved in electric vehicle applications. Electric vehicle applications typically involve partial discharges followed complete recharging.

There are a list of definitions given to help us better understand the definition of SOC before we introduce the estimation methods in details.

A cell is fully discharged when its voltage reaches $U=U_{L}$ after being drained at infinitesimal current levels which generally takes $0.1 \mathrm{~A}$. Here, we take $U_{L}=3.2 \mathrm{~V}$ at room temperature.

The capacity of a cell is the maximum number of ampere-hours that can be drawn from the cell at the practical rate before it is fully discharged, starting with the cell fully charged.

The remaining capacity is the number of ampere hours that can be drawn from the cell at room temperature at the $C / 30$ rate before it is fully discharged.

\section{The Estimate Model of an Amendment Ah Metrology}

SOC is mainly referred to the percentage of present restored charge of a battery, which is one of the most important parameters of battery management systems. We are able to understand the current state of the battery through SOC intuitively and quickly. $Q_{n}$ refers to nominal capacity respectively at room temperature $\left(25^{\circ} \mathrm{C}\right)$, when the discharging rate is set to the normative level $C / 30$. The Unites States Advanced Battery Consortium defined SOC as the ratio of standard remaining capacity $Q_{c}$ to the nominal capacity $Q_{n}$.

$$
S O C=\frac{Q_{c}}{Q_{n}}
$$

In this paper, the amended Ah Metrology is based on the characteristics of lithium-ion batteries, which has made SOC estimation value corrected. And the final SOC values obtained are more approximate to the true value. The estimate model of the amendment Ah Metrology is as follows:

$$
S O C=M_{t} \times \alpha \times S O C_{0}+\left(1-M_{t}\right) \times \alpha \times S O C_{k}-\frac{1}{C_{A}} \int_{t_{0}}^{t} \eta \times i(t) d t
$$


Among above, $\alpha$ denotes the corrected factor of the aging factor and selfdischarge; $S O C_{0}$ indicates the initial value of $S O C ; M_{t}$ is related to the off time; $S O C_{k}$ denotes the $S O C$ value stored in EEPROM when off time; $C_{A}$ indicates the total amount of electricity; $\eta$ denotes a charge-discharge efficiency. When the battery is in working condition, we update SOC value in real time with Ah-Metrology. When the battery system starts or exists in a short period of no-work, we apply the open-circuit voltage method to correct the initial value of SOC.

\subsection{The initial value of SOC}

By the adoption of Lithium-ion open-circuit voltage and SOC data, Fig.1 indicates relationship of the open-circuit voltage and the battery SOC. It is clear that the SOC value can be obtained by the open-circuit voltage at the current time.

Fig. 2 indicates the relationship between SOC value of the electric vehicle in the parking time and the time that battery required to reach steady state. As it is showed in the Fig.2, SOC value is inversely proportional to time, which determines whether Figure 1 could be used as the initial SOC value.

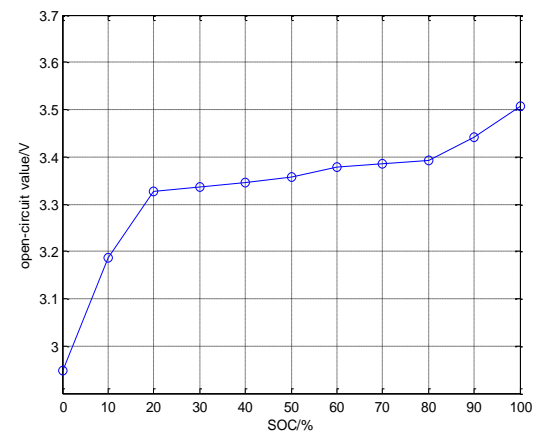

Fig. 1 The relationship between open-circuit value and SOC 


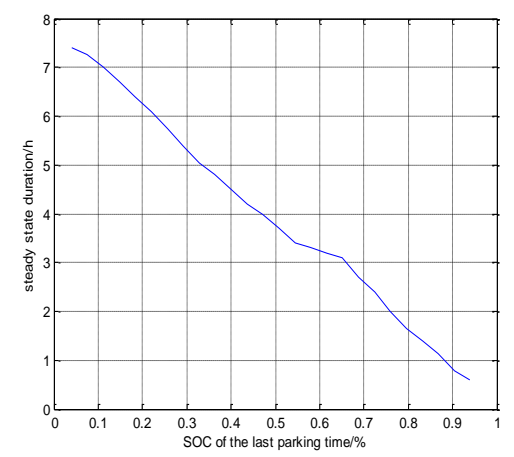

Fig. 2 The relationship between steady state duration and SOC

\subsection{Aging factors}

For lithium-ion batteries, the process of the batteries charging and discharging is a chemical reaction process. The polarization phenomenon has resulted inthe battery capacity decrease and internal resistance increase. The following is a set of aging factor $\alpha$,

$$
\alpha= \begin{cases}1 & N \leq 800 \\ 0.98 & 800 \leq N \leq 2000 \\ 0.95 & N \geq 2000\end{cases}
$$

Where $N$ and $\alpha$ are the number of charging, discharging and the lithium battery aging coefficient, respectively.

\subsection{Charging and discharging efficiency}

When the battery discharged with a differnt current, the discharging efficiency at this time is different and the power is different. The famous Peukert formula shows,

$$
I^{n} K=T
$$

Where $I$ is discharge current; $T$ is discharge time; $n$ represents constant for different types of electric vehicle battery; $K$ is constant for the active materials.

The discharge efficiency of electric vehicle battery has relationship with dishcharge current and power. So we have,

$$
Q_{n}=I_{n}^{1-n} K
$$

and 


$$
\frac{Q}{Q_{n}}=\frac{I^{1-n} K}{I_{n}^{1-n} K}
$$

We have defined that

$$
\eta=\frac{I^{1-n}}{I_{n}^{1-n}}
$$

So we have got

$$
Q=\eta Q_{n}
$$

In this paper, by the adoption of capacity and current data at room temperature, the data is fitting with the method of least squares and the relationship is obtained between the efficiency and the current,

$$
\eta=0.0007 I^{2}-0.0091 I+1.0031
$$

\subsection{Battery capacity}

Lithium-ion battery capacity is affected by the ambient temperature, so the algorithm must consider the effects of the temperature. By the adoption of Lithium-ion capacity and temperature data [8, 9], we get Fig.3. Fig.3 indicates relationship between the ambient temperature and battery capacity. As showed in Fig.3, the capacity also increases with the increase of temperature.

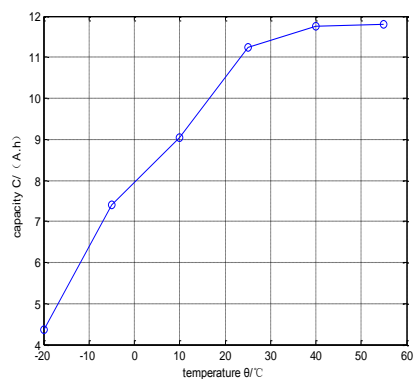

Fig. 3 The relationship between capacity and temperature

\subsection{The amended Ah algorithm}




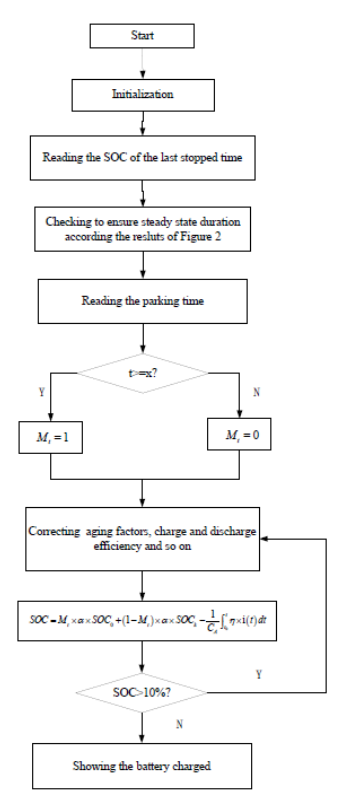

Fig. 4. The amended Ah-Metrology

Fig.4 indicates the improved algorithm of Ah integral method. It is clear that if the parking time is larger than steady state duration, the initial value of SOC could be determined by the open-circuit voltage. Otherwise, the initial value of SOC is determined by SOC of the last stopped time.

\section{Experimental Results and Analysis}

In order to illustrate the effectiveness of our proposed method, we present performance comparison between the propsed amended Ah method and the traditional Ah method.

When the discharge rate is $1 \mathrm{C}$ and the cut-off discharge voltage is $3.2 \mathrm{~V}$, simulation results are given for above algorithm implemented. The simulation results are shown in Fig.5.

The experimental results show that the estimation value of the initial SOC is 97.4\% by using traditional Ah method, while with the improved Ah integral method is $98.3 \%$. In discharge cut-off state, the SOC value is $12 \%$, while with the traditional Ah integral method is $4.8 \%$, so the error is $7.2 \%$. And with the improved amended Ah method SOC is $13.3 \%$, the error is $1.3 \%$. Hence, the proposed Ah method has advantages to improve SOC estimation accuracy. 


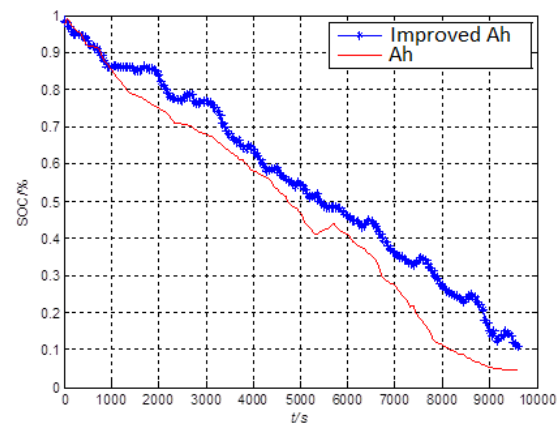

Fig. 5 Comparison of SOC estimation between the traditional Ah-Metrology and the amended Ah Metrology

\section{Conclusion}

SOC of the battery is one of the most important parameters in the use process of battery. At the same time, the estimation accuracy of SOC has great significance to economy and security of electric vehicles. This study investigated the improvement of SOC estimation. A amended Ah model was studied. Through analyzing the relevant parameters of affecting SOC estimation value, charging and discharging efficiency, aging factors and temerature parameters and corrected the SOC initial value were considered to realize the accurate estimation of SOC. Through amending the relate factors of Ah, the paper showed that the amended Ah could eliminate the accumulated error and improve SOC estimation accuracy.

\section{Acknowledgments}

This work was supported by the National Natural Science Foundation of China under Grant No.61104005 and 61374097, but Natural Science Foundation of Liaoning Province under Grant NO.201202073, and by the Open Research Fund from the State Key Laboratory of Rolling and Automation, Northeastern University, Grant No. 2015001.

\section{References}

1. Rezaee S, Farjah E, KhorramdelB. Probabilistic Analysis of Plug-in Electric Vehicles Impact on Electrical Grid through Homes and Parking Lots. IEEE Transactions on Sustainable Energy, 2013, 4(4):1024-1033. 
2. TakahashiK K, Tsujikawa T, Hirose K. Estimating the Life of Stationary Lithium-ion Batteries in Use through Charge and Discharge Testing. Telecommunications Energy Conference, Vancouver, 2014: 1-4.

3. Pei L, Lu R, Zhu C. Relaxation Model of the Open-circuit Voltage for State-of-charge Estimation in Lithium-ion Batteries. IET Electrical Systems in Transportation, 2013,3(4):112-117.

4. Xiong R, He H, Sun F, Zhao K. Evaluation on State of Charge Estimation of Batteries with Adaptive Extended Kalman Filter by Experiment Approach. IEEE Transactions on Vehicular Technology, 2013, 62(1): 108117.

5. Qiao L, Wang J, Zheng B. An Improved SOC Algorithm for Lithium-ion Batteries in Electric Vehicles. Electronics Information and Emergency Communication,Beijing, 2013:313-316.

6. Partovibakhsh M, Liu G. An Adaptive Unscented Kalman Filtering Approach for Online Estimation of Model Parameters and State-of-Charge of Lithium-ion Batteries for Autonomous Mobile Robots. IEEE Transactions on Control Systems Technology, 2015,23(1):357-363.

7. Corno M, Bhatt N, Savaresi S M, Verhaegen M. Electrochemical ModelBased State of Charge Estimation for Li-ion Cells. IEEE Transactions on Control Systems Technology, 2015,23(1):117-127.

8. Luo L, Song W, Lin S, et al. Research Progress on Effects of Temperature on SOC and Its Estimation for LFP Battery. Advances New and Renewable Energy, 2015,3(1):59-69.

9. Feng F, Lu R, Zhu C. State of Charge Estimation of Li-Ion Battery at Low Temperature. Transactions of China Electrotechnical Society, 2014, 29(7):53-59.

10. Plett GL. Extended Kalman Filtering for Battery Management Systems of LiPB-based HEV BatteryPacks: Part 2. Modeling and identification. J.Power Sources, 2004, 134(2): 262-276.

11. Tang K M, Sun L, Chan WL. Identification and Modeling of Lithium-ion battery. Energy Convers.Manag.,2010,51(12):2857-2862.

12. He H, Xiong R, Fan J. Evaluation of Lithium-ion Battery Equivalent Circuit Models for State of Charge Estimation by an Experimental Approach. Energies, 2011,4(4):582-598.

13. Xu J, Mi C, Cao B, Cao J. A New Method to Estimate State of Charge of Li-ion Batteries Based on the Battery Impedance Model. J.Power Sources, 2013,233:277-284. 